\title{
A stray light correction analysis framework for the calibration of Sentinel-4/UVN
}

Ljubiša Babic, P. Foglia Manzillo, M. Esposito, E. Loots, A. Ludewig, et al.

Ljubiša Babic, P. Foglia Manzillo, M. Esposito, E. Loots, A. Ludewig, Q. Kleipool, S. Grabarnik, F. Bernard, G. Bazalgette Courrèges-Lacoste, "A stray light correction analysis framework for the calibration of Sentinel-4/UVN," Proc. SPIE 11852, International Conference on Space Optics — ICSO 2020, 1185235 (11 June 2021); doi: 10.1117/12.2599532

SPIE Event: International Conference on Space Optics - ICSO 2021, 2021, Online Only 


\section{International Conference on Space Optics-ICSO 2020}

Virtual Conference

30 March-2 April 2021

Edited by Bruno Cugny, Zoran Sodnik, and Nikos Karafolas
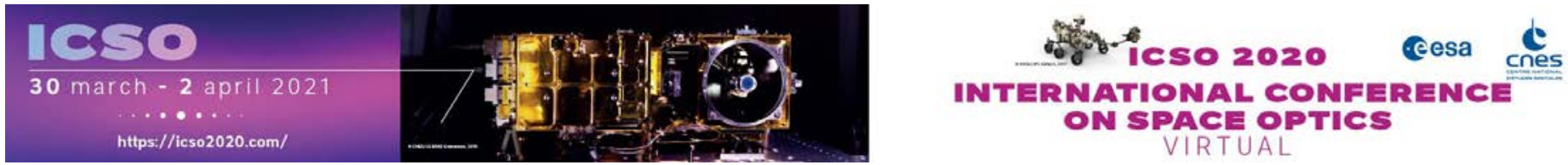

\section{A stray light correction analysis framework for the calibration of Sentinel-4/UVN}

\section{Cesa isoporeseatings denes}




\title{
A stray light correction analysis framework for the calibration of Sentinel-4/UVN
}

\author{
L. Babić*a , P. Foglia Manzillo ${ }^{\mathrm{a}}$, M. Esposito ${ }^{\mathrm{a}}$, E. Loots ${ }^{\mathrm{b}}$, A. Ludewig ${ }^{\mathrm{b}}$, Q. Kleipool ${ }^{\mathrm{b}}, \mathrm{S}$. Grabarnik ${ }^{\mathrm{c}}$, \\ F. Bernard ${ }^{c}, \mathrm{G}$. Bazalgette Courrèges-Lacoste ${ }^{\mathrm{c}}$ \\ a cosine measurement systems BV, Oosteinde 36, 2361 HE Warmond, The Netherlands; ${ }^{b}$ The Royal \\ Netherlands Meteorological Institute (KNMI), PO Box 201, NL-3730 AE De Bilt, The Netherlands; \\ 'European Space Agency, ESTEC, Postbus 299, 2200 AG Noordwijk, The Netherlands
}

\begin{abstract}
Sentinel-4/UVN is a high resolution UVN (UV-VIS-NIR) spectrometer system, being developed within the framework of the Copernicus programme, to support operational monitoring of air quality and climate over Europe and adjacent regions, through accurate atmospheric measurements of trace gas concentrations and aerosols. Stray light is one of the main drivers of the instrument performance. We have developed a stray light correction analysis framework, applicable to a wide range of spectrometer systems, to provide input for selecting the right correction method(s) and specifying calibration measurements, well before the on-ground calibration campaign. This was accomplished by using the instrument design parameters and simulated instrument response to evaluate the S4-UVN stray light performance, before and after stray light correction.

This paper presents the developed framework and a stray light correction sensitivity analysis performed for Sentinel-4/UVN. The analysis results indicate that an iterative convolution correction algorithm can reduce stray light levels in case of smooth stray light, such as diffraction and scattering. The observed fast convergence of the algorithm suggests that a single iteration may suffice in many cases. The study shows that calibration uncertainties should be carefully considered during the preparation for the on-ground calibration campaign, as they can significantly degrade the correction quality.
\end{abstract}

Keywords: Copernicus programme, Sentinel-4, spectrometer, on-ground calibration, stray light, stray light calibration, stray light correction

\section{INTRODUCTION}

Sentinel-4/UVN is a high resolution UVN (UV-VIS-NIR) spectrometer system, dedicated to providing timely and accurate measurements of Earth's atmospheric composition over Europe and North Africa, in support of operational services covering air quality and climate monitoring ${ }^{1}$. Unlike already flown instruments of this class (SCIAMACHY, OMI, GOME, and GOME 2) and those spectrometers recently launched (TROPOMI) or currently under development (Sentinel-5), S4-UVN will collect data from a geostationary orbit with a high revisit time of 60 minutes. The increased frequency of cloud-free observations will enable the assessment of the troposphere variability.

In case of high performance, high resolution spectrometer systems, such as Sentinel-4/UVN ${ }^{2}$, stray light is usually one of the main drivers of the instrument performance. Although efforts are typically made during optical design to sufficiently reduce stray light levels by using smooth surfaces, anti-reflection coatings, and baffles, the raw detector signal may still contain more stray light than it is acceptable. If the stray light response of the instrument is known, by means of characterization measurements during an on-ground calibration campaign, it is possible to further reduce the instrument stray light by processing raw data with an appropriate stray light correction algorithm.

*l.babic@cosine.nl; www.cosine.nl 
In order to sufficiently reduce stray light levels, not only the right correction method has to be selected, but also the calibration measurements have to be of sufficient quality. Preliminary selection of the correction method and specification of the calibration measurements are preferably done well before the on-ground calibration campaign, in the stage of the project when the experimental data on the stray light response of the instrument are usually not available.

To provide timely input for the S4-UVN on-ground calibration campaign, we used the instrument design parameters and simulated instrument response to evaluate the instrument stray light performance, before and after stray light correction. This was done with a stray light correction analysis framework, developed by considerably adjusting and expanding a software framework, originally created at KNMI to perform a very similar analysis for FLORIS. The S4-UVN study assessed the effectiveness of the iterative convolution correction method developed for the stray light correction of $\mathrm{TROPOMI}^{3,4}$. The impact of calibration uncertainties on the correction quality was also investigated.

The paper first describes the stray light correction analysis framework and briefly comments on its applicability to a wide range of spectrometers. The S4-UVN study is then discussed in detail. The last section provides a summary and conclusions, as well as a short outlook.

\section{STRAY LIGHT CORRECTION ANALYSIS FRAMEWORK}

A block diagram of the developed stray light correction analysis framework is shown in Figure 1 . As can be seen, the framework consists of three models: a forward model, a calibration model, and a stray light correction (reverse) model. In a nutshell, the forward model computes the signal including stray light to evaluate performance before correction, the calibration model simulates the uncertainties in characterizing the instrument stray light response, and the reverse model assesses the effectiveness of a stray light correction method by taking into account the simulated calibration uncertainties. Below we briefly discuss each model and the framework applicability.

\subsection{Forward model}

The forward model relates the at-aperture spectral radiance to the detector output expressed in electrons. This model is used to calculate the detector signal without stray light $\left(\mathrm{S}_{\mathrm{w} / \mathrm{o} \mathrm{SL}}\right)$ and the detector signal with stray light $\left(\mathrm{S}_{\text {with } \mathrm{SL}}\right)$. The stray

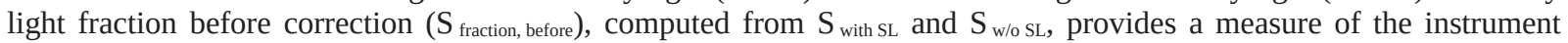
stray light performance. This information can be used to evaluate compliance with stray light requirements and to serve as input for the detailed instrument design.

In addition to the at-aperture spectral radiance, the computation of $\mathrm{S}_{\mathrm{w} / \mathrm{s}} \mathrm{s}$ requires knowledge about the etendue and throughput of the optical system, the detector quantum efficiency, the spectral width of the detector pixel, the integration time, and the number of temporally co-added signals. The instrument spectral map, relating the detector pixel number to the corresponding wavelength bin, also needs to be provided as input to the model. The finite spectral resolution of the instrument is described by the instrument spectral response function (ISRF).

A separate function, referred to as the stray light response function (SLRF) or the stray light kernel, is used to describe the response of the instrument to stray light. One way to explain the stray light response function, is to first discuss the instrument response without stray light, i.e. the response of the instrument to light that follows the path intended by the optical design, which we will refer to as the intended, wanted, or direct light. Neglecting stray light (including diffraction), only a handful of detector pixels are illuminated with a collimated and monochromatic beam in case of a diffraction limited system. The detector region illuminated by the direct light is referred to as to the direct region. In contrast, stray light illuminates also the detector pixels outside the direct region, i.e. the stray light region. While the ISRF describes the instrument response in the direct region, the SLRF characterizes the instrument response outside the direct region. Stray light response functions are obtained from the full instrument response to the collimated and monochromatic light beams by setting the detector signal in the direct region to zero. The stray correction analysis framework accepts as input both experimental and simulated SLRFs.

A stray light response function can be used to describe diffraction, scattering, ghosting, and stray light caused by unwanted grating orders. The current framework does not take into account the out-of-field-of-view stray light (detector signal caused by light outside the spectrometer field of view) and out-of-band stray light (detector signal caused by light outside the spectrometer spectral range). These effects could be included in a future version of the framework.

In order to compute $S_{\text {with SL, }}$ it is necessary to calculate the stray light signal $\left(\mathrm{S}_{\mathrm{SL}}\right)$. In its simplest form, the stray light signal computation is defined as the convolution between $\mathrm{S}_{\mathrm{w} / \mathrm{SL}}$ and the forward model stray light response function 
$\left(\mathrm{SLRF}_{\mathrm{fwd}}\right)$. In practice, the stray light evaluation involves multiple convolutions as multiple stray light response functions are needed to describe the wavelength and or field dependence of the stray light response. The developed stray light correction analysis framework supports this multiple-kernel forward model.

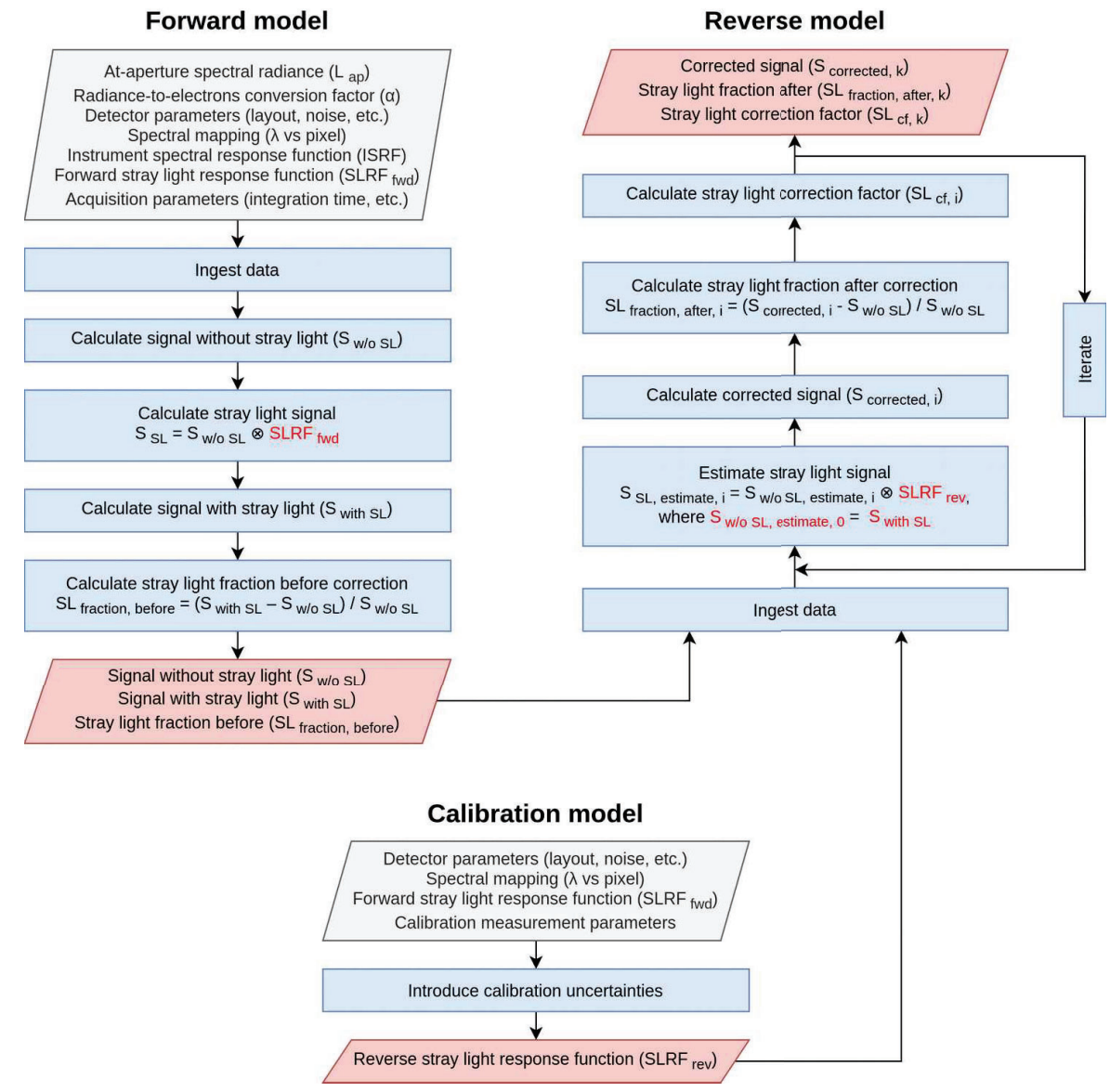

Figure 1. A block diagram of the developed stray light correction analysis framework. The top left and the top right side of the figure show the forward and reverse model, respectively. The forward model converts the at-aperture radiance into a detector signal without stray light and a detector signal with stray light. The reverse model applies an iterative convolution correction algorithm to correct for the instrument stray light simulated in the forward model. The effectiveness of the stray light correction model is quantified by the reduction in the stray light fraction. The quality of the correction is negatively affected by the difference between the forward and reverse stray light response function. This difference is simulated by the calibration model shown in the bottom of the figure.

\subsection{Reverse model}

The reverse model corrects $S_{\text {with SL }}$ for stray light. The corrected signal $\left(\mathrm{S}_{\text {corrected }}\right)$ is used to calculate the stray light fraction after correction $\left(\mathrm{S}_{\text {fraction, after }}\right)$. The stray light correction factor $\left(\mathrm{SL}_{\mathrm{cf}}\right)$, defined as the ratio between $\mathrm{S}_{\text {fraction, after }}$ and

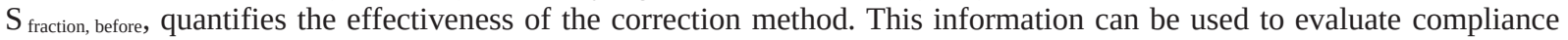
with stray light requirements applicable to the data corrected for stray light, and to serve as input for the final design of the stray light correction algorithm approach.

The current framework has only one stray light correction algorithm, referred to as the iterative stray light convolution algorithm. The central step of the convolution correction approach is estimating the stray light signal by convolving the best available approximation of $\mathrm{S}_{\mathrm{w} / \mathrm{SL}}$ and the reverse model stray light response function (SLRF rev). In the first iteration, $\mathrm{S}_{\text {with SL }}$ is used as the best guess for $\mathrm{S}_{\text {w/o SL. }}$. Multiple convolution iterations can be performed with the aim to improve the stray light correction effectiveness. The stray light correction algorithm can also correct for stray light that 
exhibits wavelength and or field dependence. In that case, multiple reverse kernels are provided as input to the convolution correction algorithm.

The reverse model is meant to be used as a test bed for a variety of correction strategies and algorithms. Therefore, more stray light correction algorithms could be added as part of the further development of the stray light correction analysis framework.

\subsection{Calibration model}

Note that the framework distinguishes between the forward and reverse model stray light kernels. The simulated forward kernels, which in principle require a perfect knowledge of the instrument stray light response, are used by the forward model to generate the stray light levels in the uncorrected signal. The simulated reverse kernels, which model the instrument stray light response, as derived from the on-ground calibration measurements, are used by the correction algorithm. The mismatch between the two degrades the correction performance. The calibration model simulates this mismatch.

The calibration model computes the reverse kernels from the forward kernels by taking into account calibration uncertainties. The current version of the model can simulate the following effects:

- $\quad$ reducing the kernel size

- neglecting the stray light dependence on the wavelength and or field

- measurement noise.

Dimensions of a stray light kernel are ideally twice as large as those of the detector, to account for all the stray light contributions. We will refer to these kernels as to full size kernels. Accordingly, all forward kernels in the stray light correction framework are full size kernels. In practice, stray light kernels are determined by measuring the instrument response to tunable lasers and/or white light sources with optical bandpass filters. This limits the size of experimentally retrieved kernels to that of the detector. It is possible to construct an even larger stray light kernel by modelling the measured stray light response. However, this might introduce a larger uncertainty in the stray light correction than using a truncated stray light kernel. The calibration model can produce reverse kernels with a reduced size by truncating full size forward kernels.

In general, the stray light response of a spectrometer system depends on both the wavelength and angle of the impinging electromagnetic radiation. The forward model takes this into account by employing multiple forward stray light kernels. The number of the used forward kernels is not limited by the forward model. Instead, it typically depends on how fast the stray light response changes as a function of the wavelength and field. In contrast, the on-ground calibration campaign imposes limitations on the number of measured stray light kernels due to time constraints. The calibration model can simulate this effect by defining the reverse kernels as a subset of the forward kernels.

The calibration model can include the measurement noise in the reverse stray light kernels. The current measurement noise model is rather simple. It has two noise components that are assumed to be random and independent. One component is the photon noise. The other component describes the combined effect of the read noise, electronics noise, electromagnetic compatibility noise, quantization noise, and dark current noise. This component is characterized by a RMS noise parameter $\beta$ that has a constant value. The total noise level is computed by adding the two noise components in quadrature. In addition, the total noise level scales down with the square root of the number of measurement repetitions.

In order to compute the photon noise in the reverse stray light kernel, it is necessary to consider a scenario for measuring the instrument response to stray light. To this end, the current version of the calibration model assumes a relatively simple measurement scenario, in which the stray light kernels are measured by uniformly illuminating regions on the detector with the size equal to the direct region size. For the sake of maximizing the SNR, the signal in the direct region reaches $90 \%$ of the detector saturation level. The stray light signal and the corresponding photon noise, are then accordingly scaled.

Developing a more complex measurement noise model and adding more calibration measurement scenarios could be considered for future versions of the framework. The impact of the gain and offset uncertainty could also be added to the calibration model, as well as the uncertainty associated with the stray light response function retrieval algorithm(s). 


\subsection{Framework applicability}

The described stray light correction analysis framework is applicable to a wide range of spectrometer systems. The framework can be used to analyse point as well as imaging spectrometers, with the latter case requiring a two-dimensional detector. Note that the framework does not specify the source of the at-aperture radiance. In this sense, the analysis framework is equally applicable to both ground and space-based spectrometers. The presented framework does not pose any requirements on the mass and volume of the system, and can therefore be used in the context of small and big satellites.

\section{SENTINEL-4/UVN CASE STUDY}

We performed a stray light correction analysis for the S4-UVN instrument with the above described framework. In this section, we discuss this case study. We first list the main system parameters and introduce the applicable stray light reference scene. Then, we present the investigated stray light correction analysis cases. At the end, we show and discuss the results of the analysis.

\subsection{Main system parameters and stray light reference scene}

Table 1 summarizes the main parameters of the S4-UVN spectrometer system consisting of a UVVIS and NIR spectrometer. Note that the UVVIS detector is divided into two image zones denoted as UVVIS1 and UVVIS2. Although the two image zones are read out independently and can have different integration times, their combined output was considered as a single detector image for the purpose of the stray light correction analysis.

Table 1. An overview of the main parameters of the S4-UVN instrument.

\begin{tabular}{|c|c|c|}
\hline Parameter & UVVIS spectrometer & NIR spectrometer \\
\hline Spectral range $[\mathrm{nm}]$ & $\begin{array}{l}\text { 305-340 (UVVIS1) } \\
340-500 \text { (UVVIS2) }\end{array}$ & $750-775$ \\
\hline Spectral resolution [nm] & 0.5 & 0.12 \\
\hline Focal length [mm] & 214.95 & 214.95 \\
\hline Field of view $\left[{ }^{\circ}\right]$ & 4 & 4 \\
\hline f-number & 3.05 & 4.93 \\
\hline Slit width $[\mu \mathrm{m}]$ & 44.3 & 44.3 \\
\hline Slit length [mm] & 15.02 & 15.02 \\
\hline Magnification & 1.0 & 1.0 \\
\hline Detector pixel size & $27.5 \mu \mathrm{m}$ (spatial) $\times 15 \mu \mathrm{m}$ (spectral) & $27.5 \mu \mathrm{m}$ (spatial) $\times 15 \mu \mathrm{m}$ (spectral) \\
\hline Detector format & 580 px (spatial) x 1274 (spectral) px & 580 px (spatial) x 704 (spectral) px \\
\hline $\begin{array}{l}\text { Detector integration time } \\
\text { [ms] }\end{array}$ & $\begin{array}{l}258.6 \text { (UVVIS1) } \\
129.3 \text { (UVVIS2) }\end{array}$ & 517.2 \\
\hline $\begin{array}{l}\text { Number of co-added } \\
\text { images }\end{array}$ & $\begin{array}{l}24 \text { (UVVIS1) } \\
48 \text { (UVVIS2) }\end{array}$ & 12 \\
\hline Detector saturation level & $1.35 \mathrm{Me}^{-}$ & $0.88 \mathrm{Me}^{-}$ \\
\hline
\end{tabular}

Figure 2 shows a reference scene applicable for assessing the stray light performance of the S4-UVN instrument. The

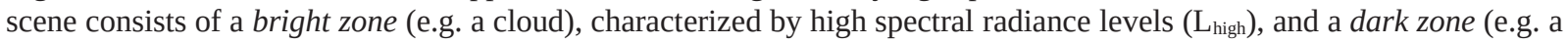
reference target), characterized by low spectral radiance levels $\left(\mathrm{L}_{\text {low }}\right)$. The instrument stray light performance is evaluated 
in the dark zone at a distance larger than 10 SSD (spatial sampling distance) from the edge of a transition between the bright and dark zone.

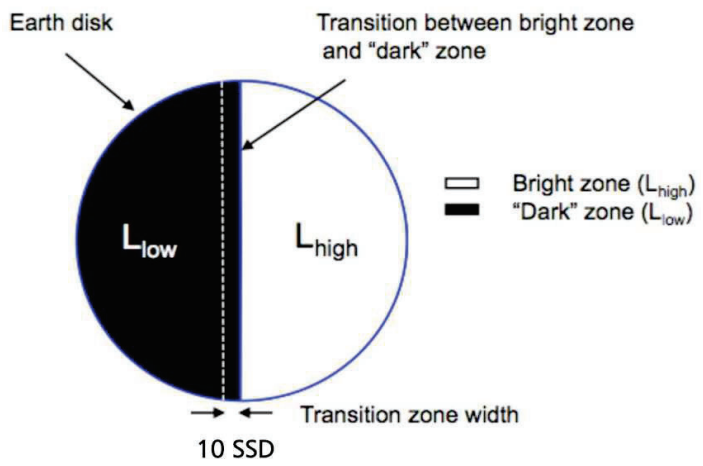

Figure 2. The stray light reference scene for the S4-UVN instrument consisting of a bright and dark zone. The stray light performance is assessed in the dark zone at a distance larger than 10 SSD from the edge of a transition between the bright (e.g. a cloud) and dark zone (e.g. a reference target).

\subsection{Stray light correction analysis cases}

Four stray light correction analysis cases were investigated in the S4-UVN instrument study. All the cases shared the same forward model, but they differed in the reverse kernels that were employed in the correction (reverse) model. Here, we first discuss the used forward model with an emphasis on the forward stray light kernels. Then, we address the differences between the sets of reverse stray light kernels.

To take into account the wavelength dependence of the stray light response, we used a multiple-kernel forward model for both the UVVIS and NIR spectrometer. The UVVIS forward model was constructed using nine wavelength dependent stray light kernels, calculated at 300, 325, 350, 375, 400, 425, 450, 475, and $500 \mathrm{~nm}$. Due to a much narrower spectral range, the NIR forward model featured only two wavelength dependent stray light kernels, one calculated at $756.25 \mathrm{~nm}$ and the other at $768.75 \mathrm{~nm}$.

Stray light kernels were calculated as a sum of the scattering and diffraction contribution. The scattering contribution, caused by the surface roughness and particulate contamination of both the spectrometer and telescope, was calculated with StrayLux ${ }^{5,6}$, an efficient stray-light modelling tool based on analytical expressions for in-field scattered light distributions ${ }^{7}$. The diffraction contribution was computed from the f-numbers reported in Table 1, as the intensity of the Fraunhofer diffraction pattern of a circular aperture.

Each forward kernel was computed as a full size kernel, i.e. each kernel dimension was twice the corresponding detector dimension. The direct region, where the stray light response was set to zero, extended nine and five pixels in the spectral and spatial direction, respectively. Furthermore, the forward stray light kernels were not affected by the measurement noise. Examples of simulated full size, noiseless stray light kernels are shown in Figure 3. The kernel rows (spatial direction) are displayed vertically and the kernel columns (spectral direction) are displayed horizontally.

The calibration model was used to introduce uncertainties in the reverse stray light kernels. These uncertainties were propagated by the reverse model to evaluate their impact on the stray light correction factor. Based on the differences in the used reverse stray light kernels, we distinguish between the following four correction cases:

- Case 1: Ideal case (limit of the iterative convolution approach)

- Reverse kernels are identical to the forward kernels.

- Case 2: Reverse kernels with a reduced size (impact of reducing the kernel size)

- Except for their size, the reverse kernels are identical to the forward kernels.

- The size of each reverse kernel is equal to the detector size.

- Case 3: Single reverse kernel with a reduced size (additional impact of neglecting the stray light wavelength dependence) 
- The correction is performed with a single, detector size reverse kernel, without taking into account the measurement noise.

- Reverse kernels computed at 400 and $762.5 \mathrm{~nm}$ are used for the UVVIS and NIR spectrometer, respectively. The reverse kernels are shown in the middle panels of Figure 4.

- $\quad$ Case 4: Noisy single reverse kernel with a reduced size (additional impact of the measurement noise)

- The same reverse kernels are used as in the case above, but with the measurements noise included.

- Four measurement noise levels are considered: no noise and three noise levels corresponding to 1000000 , 10 000, and 100 repeated measurements. The RMS noise parameter $\beta$ is respectively 72 and 44 electrons, for the UVVIS and NIR spectrometer. The reverse kernels corresponding to the case with the highest noise level are shown in the bottom panels of Figure 4.
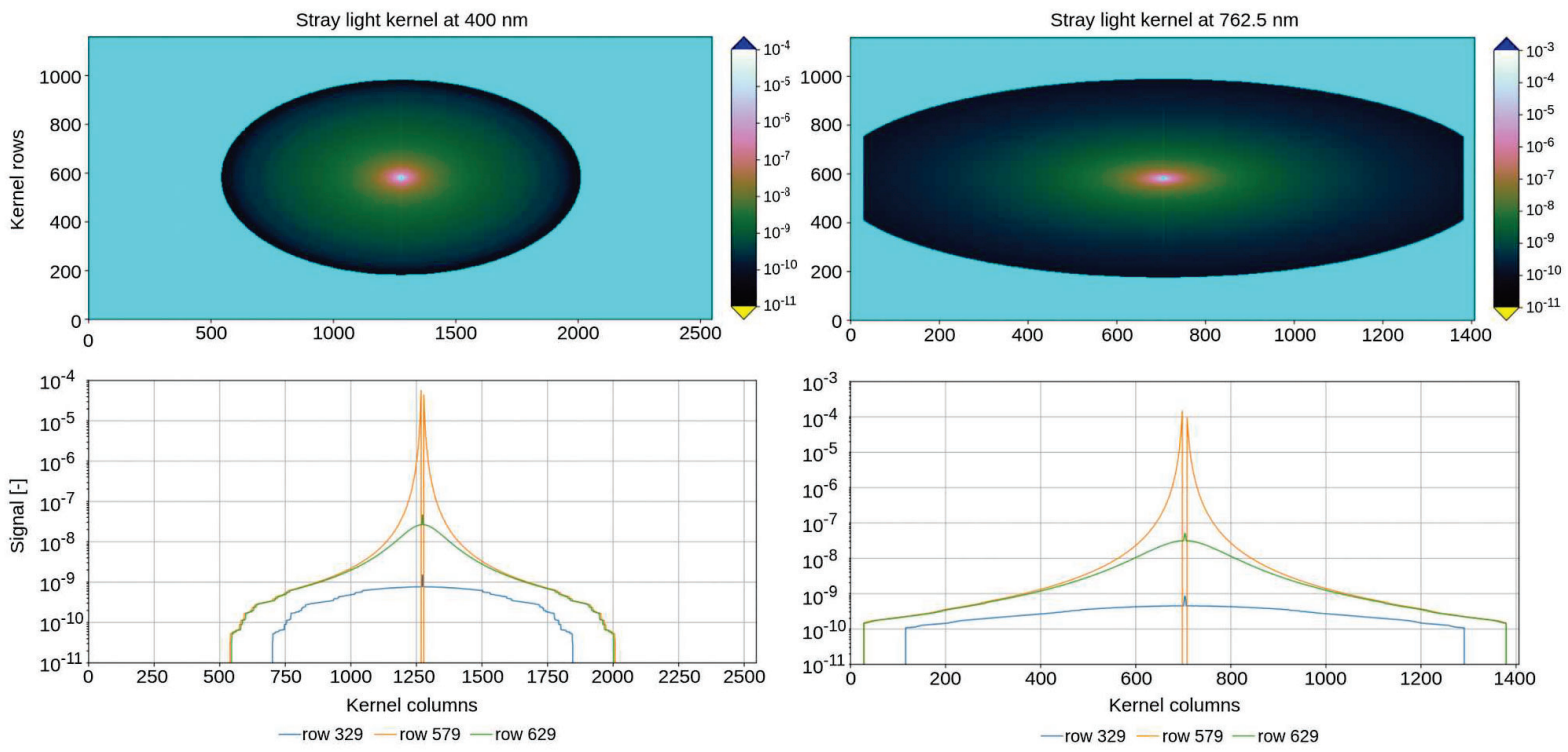

Figure 3. A full size stray light kernel without measurement noise, simulated at 400 (left) and $762.5 \mathrm{~nm}$ (right). (Top panels) The kernel image, with the kernel rows (spatial direction) depicted vertically and the kernel columns (spectral direction) depicted horizontally. The cyan colour in the image denotes the zero-valued elements of the kernel. (Bottom panels) Cross-sections at three kernel rows including the central kernel row 579. The stray light response is zero in the direct region extending nine and five pixels in the spectral and spatial direction, respectively. The presented stray light kernels take into account scattering (surface roughness and particulate contamination of both the spectrometer and telescope) and aperture diffraction. The total integrated signal, which includes also the direct contribution, is normalized to unity. 

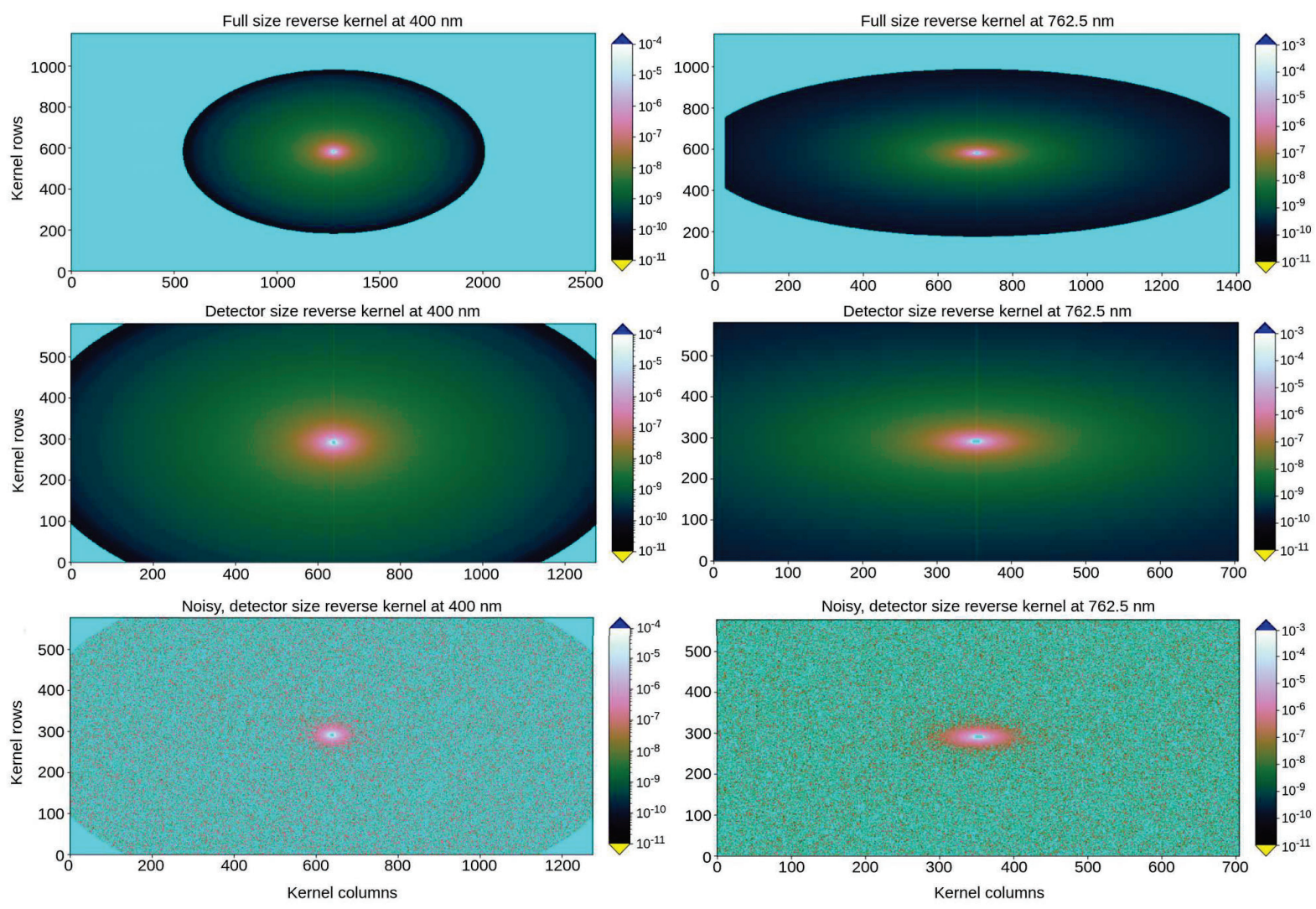

Figure 4. The impact of investigated calibration uncertainties on a reverse stray light kernel, simulated at 400 (left) and $762.5 \mathrm{~nm}$ (right). The kernel rows (spatial direction) are depicted vertically and the kernel columns (spectral direction) are depicted horizontally. The cyan colour denotes the elements of the kernel that are either equal to zero or negative due to measurement noise. The figure shows a transformation from a full size reverse kernel without measurement noise (top panels) first to a noiseless, detector size reverse kernel (middle panels), and then to a noisy, detector size reverse kernel simulated for 100 measurement repetitions (bottom panels). As can be seen, the kernel size reduction effectively discarded useful information about the instrument response to stray light. The measurement noise further degraded the knowledge about the stray light response. Both effects are expected to negatively affect the stray light correction effectiveness.

\subsection{Results and discussion}

Figure 5 shows a stray light reference scene without stray light, simulated for the UVVIS (left) and NIR spectrometer (right). The top panels present the resulting detector image after co-adding individual frames. The middle panels display cross-sections in the column (spectral) direction revealing spectral features of the reference scene, such as the $\mathrm{O}_{2}$-A band in the NIR. The bottom panels show cross-sections in the row (spatial) direction.

To indicate the transition between the bright and dark zone, the middle panels purposely show a cross-section at row 289 and a cross-section at row 290. The bright-to-dark transition can also be observed in the cross-sections in the row direction, i.e. in the bottom panels of the figure. The presented data provide information on the contrast between the two zones.

For each spectrometer, the stray light fraction before correction was calculated from the simulated reference scene without and with (not shown here) stray light. The results are shown in Figure 6. The left and right panels respectively present the data for the UVVIS and NIR spectrometer.

The middle panels include the cross-section at row 300, because the stray light performance was evaluated in the dark zone starting from the 11th pixel away from the transition between the bright and dark zone. As can be seen from the figure, the stray light levels decrease with increasing the distance from the transition between the two zones. 

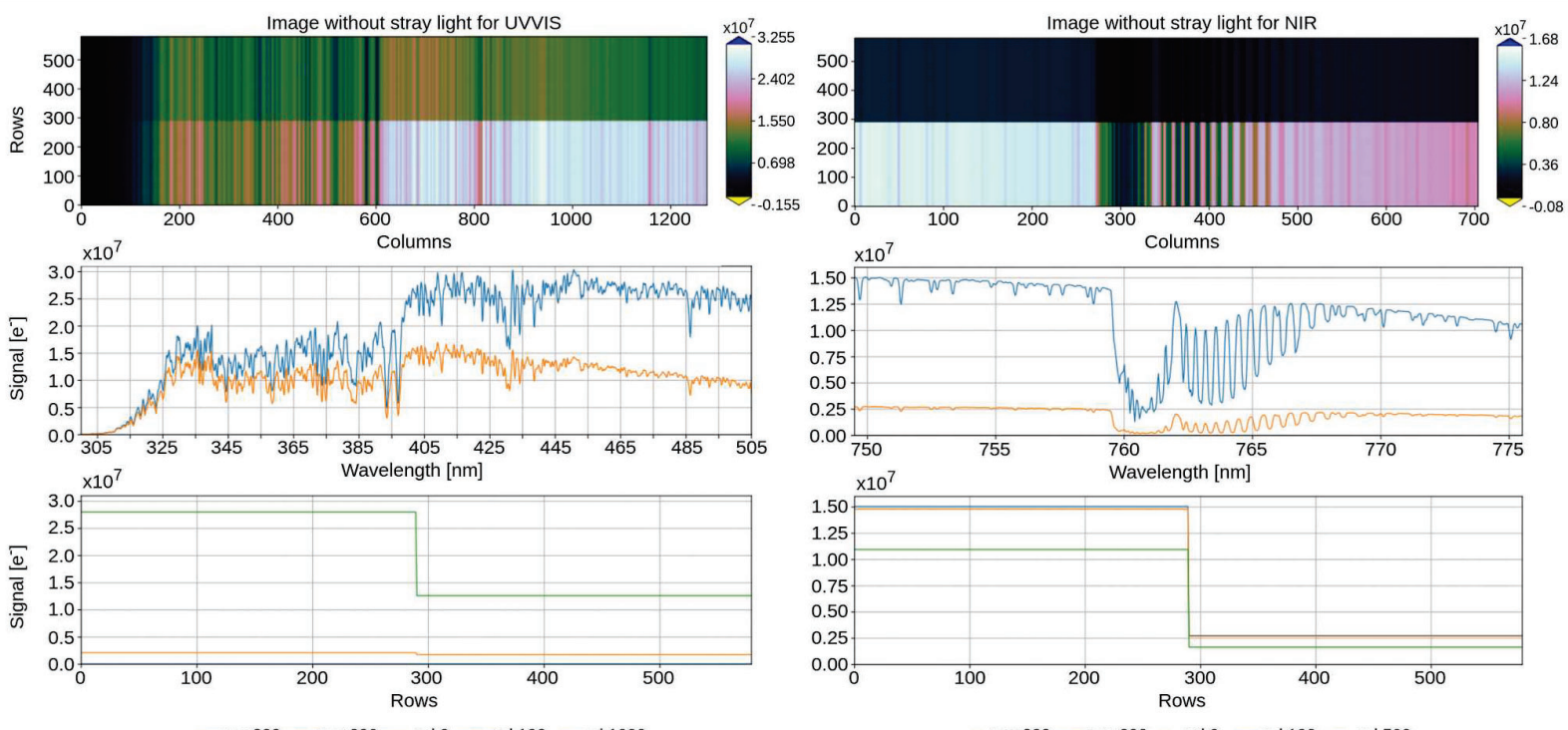

- row 289 - row $290-\operatorname{col} 0-\operatorname{col} 100-\operatorname{col} 1000$

- row $289-$ row $290-\operatorname{col} 0-\operatorname{col} 100-\operatorname{col} 500$

Figure 5. A stray light reference scene without stray light, computed for the UVVIS (left) and NIR spectrometer (right). (Top panels) The detector image, with the rows (spatial direction) depicted vertically and the columns (spectral direction) depicted horizontally. (Middle panels) Cross-sections in the column (spectral) direction revealing spectral features in the reference scene. (Bottom panels) Cross-sections in the row direction. Note the transition between the bright (row 289) and dark zone (row 290).
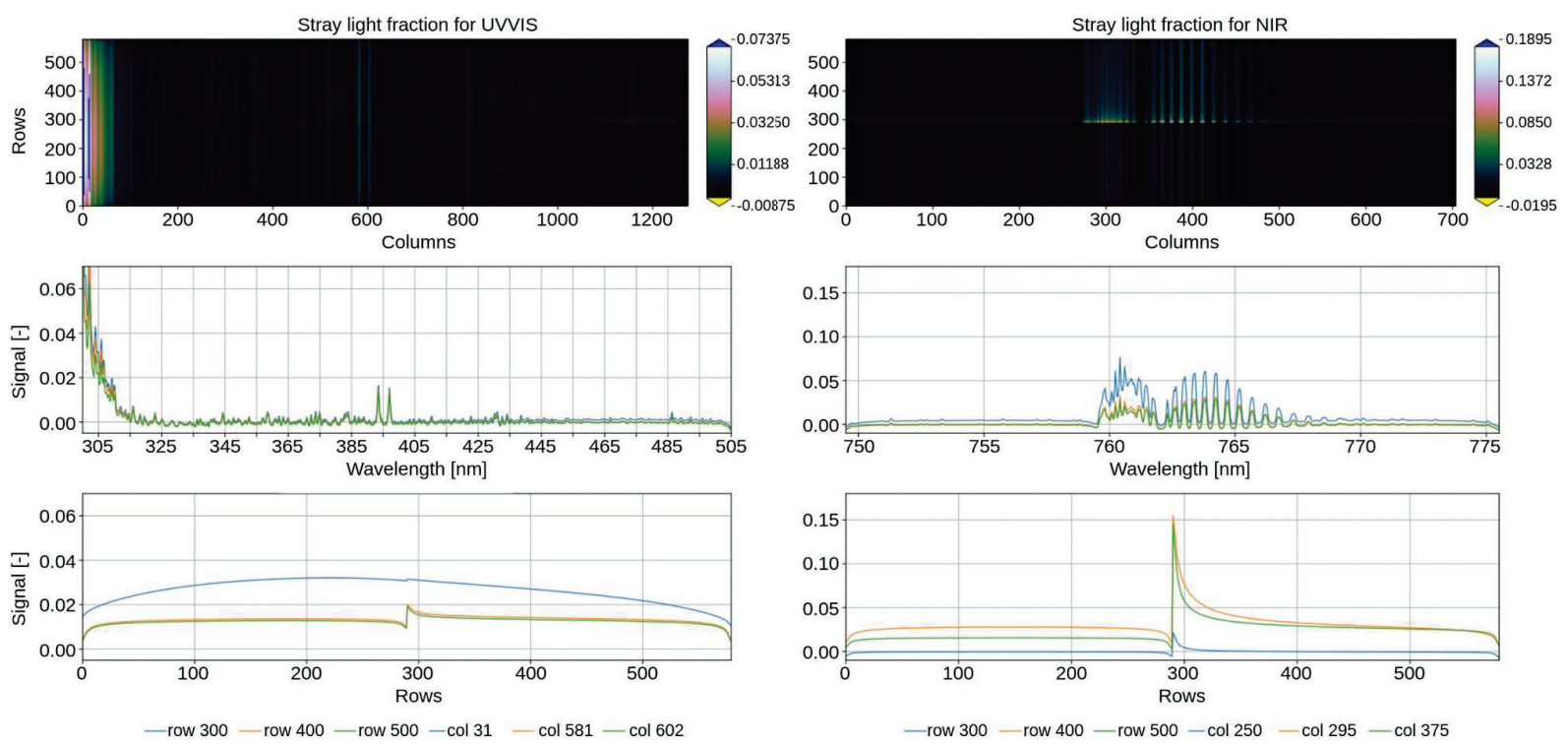

Figure 6. The stray light fraction before correction, simulated for the UVVIS (left) and NIR spectrometer (right). Note that the stray light performance was evaluated in the dark zone starting from row 300, i.e. the 11th pixel away from the transition between the bright and the dark zone. In line with expectations, the highest stray light levels were found around $305 \mathrm{~nm}$ in the UVVIS and in the O2-A band in the NIR. Stray light fractions as high as $3.7 \%$ and $7.7 \%$ were computed in the performance spectral range of the UVVIS and NIR spectrometer, respectively. 
For the UVVIS spectrometer, the computed stray light fraction was the highest in the spectral region around $305 \mathrm{~nm}$ due to a low direct signal in the UV. In addition, the stray light signal exhibited two peaks around 395 nm caused by two Fraunhofer solar absorption lines. The stray light signal reached a maximum value of $3.7 \%$ in the performance spectral range of the UVVIS spectrometer. In case of the NIR spectrometer, the calculated stray light signal was the strongest in $\mathrm{O}_{2}$-A band, as expected. The computed NIR stray light levels were as high as $7.7 \%$.

Evaluating the instrument stray light performance before correction, as presented in Figure 6, is useful in both the early and late stage of the instrument development. The early stage allows for tuning the preliminary design to reduce the stray light levels. In the late stage, the simulated stray light levels can guide estimates for the required effectiveness of the stray light correction approach.

The reverse model was used to calculate the stray light fraction after correction, for each of the four correction cases. Because the stray light fraction varies from pixel to pixel, the quality of the correction can be evaluated in a number of ways. In many cases, it is the maximum stray light level that matters the most. This approach was also adopted for the S4-UVN study presented here. The effectiveness of the stray light correction was determined as the ratio between the maximum of the absolute value of the stray light fraction before correction and the maximum of the absolute value of the stray light fraction after correction. Only the detector pixels in the applicable region of the dark zone were used for this calculation.

Figure 7 reports the computed stray light correction factor for the first three correction cases without measurement noise, showing effects of systematic uncertainties on the quality of the stray light correction. The data are shown as a function of the number of convolution iterations, for the UVVIS (left) and NIR (right) spectrometer. The correction effectiveness for the ideal case, where the reverse kernels are identical to the forward kernels, is indicated by the solid black lines. The impact of reducing the reverse kernel size is denoted by the dashed blue lines, and the effect of neglecting the stray light wavelength dependence in the reverse model is shown with the dash-dotted red lines.

A number of observations and conclusions can be drawn from the presented data. First of all, the iterative convolution approach reduced the maximum stray light in all the three cases. This suggests that the approach can be used to correct for smooth stray light, such as diffraction and ghosting. In the ideal case, increasing the number of iterations improved the correction. This trend extended beyond the third iteration. However, we chose to limit the figure output to three iterations to enable easier visual comparison with the other two cases.
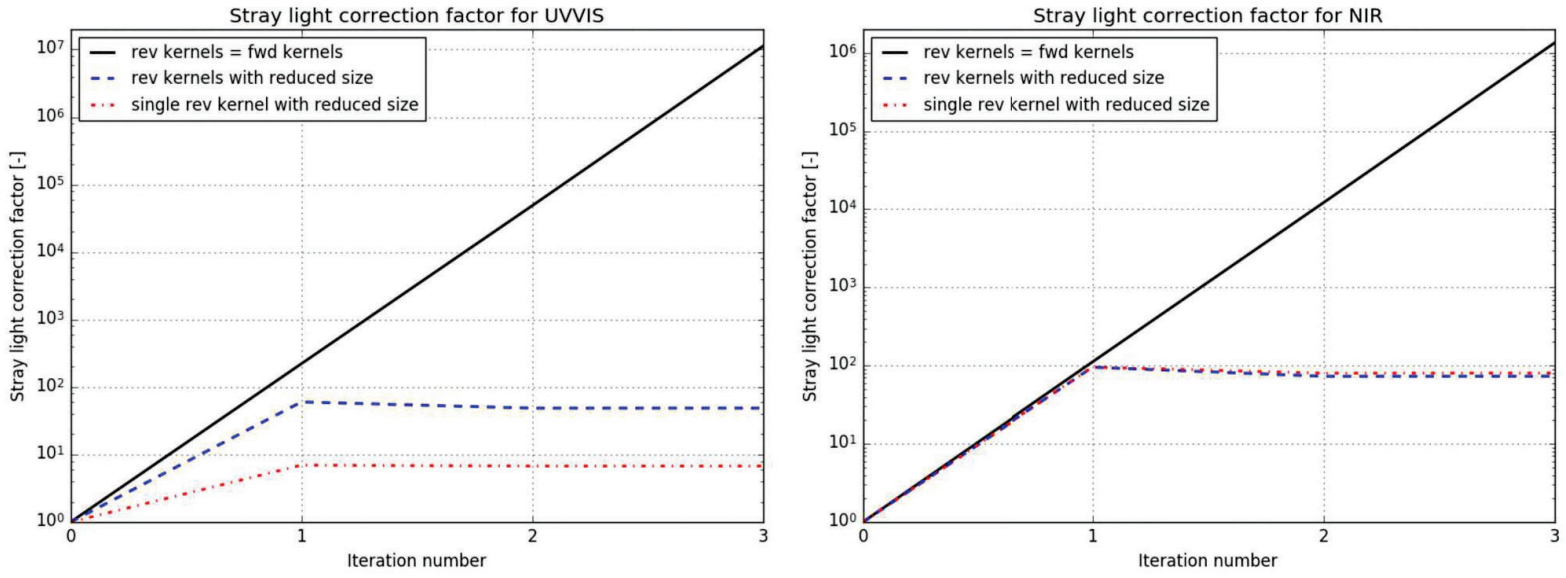

Figure 7. The stray light correction factor, computed for the UVVIS (left) and NIR (right) spectrometer, for the first three correction cases that do not take into account the measurement noise in the reverse kernel(s). The data are presented as a function of the number of convolution iterations. The solid black lines denote the ideal case, where the reverse kernels are identical to the forward kernels. The dashed blue lines show the impact of reducing the reverse kernel size, while the dash-dotted red lines show the effect of neglecting the stray light wavelength dependence in the reverse model. Clearly, the convolution algorithm reduced the stray light levels. In the ideal case, the correction factor increased with each convolution iteration. Reducing the reverse kernel size significantly degraded the quality of the correction. Whereas it further reduced the correction factor in case of the UVVIS spectrometer, neglecting the stray light dependence only marginally affected the correction factor in the NIR. 
Reducing the reverse kernel size, from twice the detector dimensions to the detector dimensions, which is expected to occur in practice, significantly degraded the quality of the correction. This was the case for both spectrometers: the correction factor was reduced by five and four orders of magnitude for the UVVIS and NIR spectrometer, respectively. In contrast, not accounting for the stray light wavelength dependence in the reverse model, had a very different impact on the individual spectrometers. The correction factor for the UVVIS was further reduced by a factor of seven. The effect on the NIR spectrometer was only marginal, likely due to a much narrower spectral range.

Finally, the data suggests that the convolution correction algorithm converges fast. In fact, the results indicate that one iteration may be sufficient in practice.

The fourth correction case built upon the third one, and explored the effect of the measurement noise in the reverse kernel. Four measurement noise levels were investigated: no noise (black), and noise levels corresponding to 10000000 (blue), 10000 (red), and 100 (green) measurement repetitions. The simulated results are shown in Figure 8. Note that it is difficult to observe the case without noise as it pretty much overlaps with the correction data for the case with the highest SNR.

The measurement noise caused a spread in the computed correction data. This is indicated by the error bars in the figure. Each error bar denotes the range between a lower and upper limit of the stray light correction factor, retrieved from 100 Monte Carlo type of simulations. In some of the Monte Carlo simulations, the measurement noise perturbed the reverse kernel in such a way to achieve even a better correction performance than in the case without the noise. This is a consequence of the statistical nature of the noise, and it does not mean that the measurement noise improves the correction. The spread in the simulation results, rather than individual simulation results, should be considered for evaluating the computed correction performance. In fact, the lower limit of a correction factor should be considered for a conservative evaluation of the simulated correction quality.

As observed from the presented spread in the stray light correction factor, the measurement noise further degraded the correction performance. Increasing the SNR narrowed the spread and brought the correction factor closer to the case without the measurement noise. These results are in line with expectations.
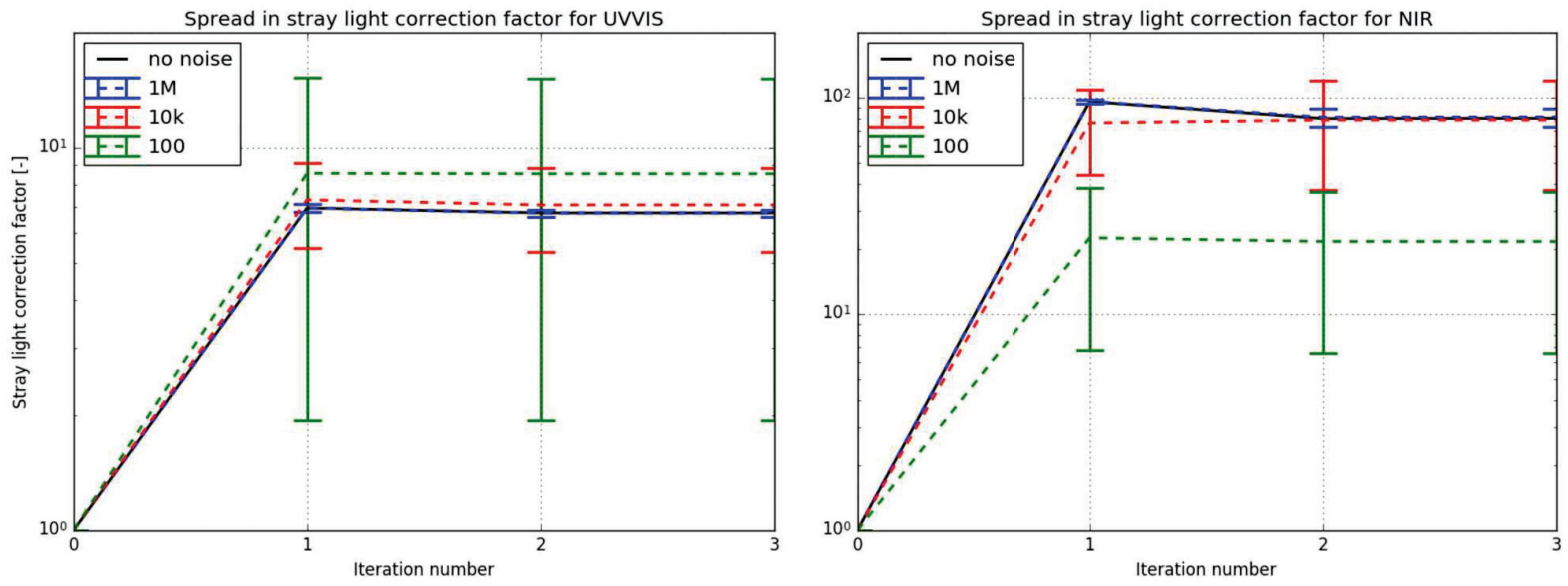

Figure 8. The impact of the measurement noise in the reverse kernel on the stray light correction factor, computed for the correction case with a single, detector-size reverse kernel. The data are presented as function of the convolution iteration number, for the UVVIS (left) and NIR (right) spectrometer. The spread in the stray light correction was simulated for four measurement noise levels: no noise (black), and noise levels corresponding to 1000000 (blue), 10000 (red), and 100 (green) measurement repetitions. The spread was determined from 100 Monte Carlo type of simulations per noise level. Each error bar denotes the range between a lower and upper limit of the stray light correction factor with a dashed line running midway (note the log scale on the y-axis) between the two limits. Note that it is difficult to observe the case without noise as it pretty much overlaps with the correction data for 1000000 repeated measurements. The results suggest that performing only 100 measurements would result in a considerably worse stray light correction, and that there would be little to gain by increasing the number of measurements beyond 1000000 . 
According to the simulation results, performing only 100 measurement repetitions would significantly reduce the correction effectiveness for both spectrometers. The data also suggests that performing more than 1000000 measurement repetitions would yield only a marginal improvement in the correction factor.

Stray light correction analysis simulations that include calibration uncertainties, like the ones show in Figures 7 and 8 , can aid the preparation for the on-ground calibration campaign. Most importantly, these simulations can provide input for choosing the appropriate strategy for stray light calibration and correction. This includes selecting and developing the most promising correction algorithm(s) and designing calibration measurements. The latter, for example, includes determining the appropriate SNR for the measurements and selecting the wavelengths at which to characterize the instrument stray light response.

\section{SUMMARY AND CONCLUSIONS}

We have developed a framework for stray light correction analysis, applicable to a wide range of spectrometer systems, including space-based spectrometers on-board large or small satellites. The framework evaluates the instrument stray light performance, before and after stray light correction. This information can be used to first aid the instrument design and later on to assist the on-ground calibration efforts.

To provide input for the on-ground calibration campaign, we performed a stray light correction analysis for the Sentinel-4/UVN instrument with the developed framework. In the absence of experimental data, the design parameter values and simulated instrument response were used as inputs. Two stray light effects were simulated in the forward model: the aperture diffraction and scattering caused by surface roughness and particulate contamination of both the spectrometer and telescope. An iterative convolution approach was employed in the reverse model to reduce the simulated stray light levels.

The study results indicate that the iterative convolution algorithm can be used to reduce stray light levels in case of smooth stray light, such as diffraction and scattering. Furthermore, the convolution algorithm was found to converge fast, suggesting that one iteration may be sufficient in many cases.

Equally important, the analysis demonstrated that calibration uncertainties can significantly degrade the correction effectiveness, and that they should be carefully considered as part of the preparation for the on-ground calibration activities. This conclusion is in line with the expectations. The added value of the framework is that it can quantify the effect of individual uncertainties on the quality of the stray light correction. The current version of the framework can evaluate the impact of the size of the reverse stray light kernels, stray light wavelength and or field dependence, and measurement noise.

Further development of the framework, potentially being carried out in parallel with the preparations for the S4-UVN on-ground calibration campaign, is anticipated to improve the fidelity of the framework by including additional stray light contributions and calibration uncertainties. Incorporating out-of-band stray light, and taking into account gain and offset uncertainty, would most likely take priority. The next step could be to expand the reverse model with an additional algorithm dedicated to correcting focused ghosts. Considering the importance of stray light mitigation for space-based spectrometers, it may be also interesting to explore the possibility of integrating the stray light correction analysis framework in end-to-end mission analysis tools, especially in the context of future Earth observation missions monitoring air quality and climate.

\section{ACKNOWLEDGEMENTS}

We would like to thank everyone involved in the development of the stray light correction analysis framework and the Sentinel-4/UVN study. We would also like to acknowledge the ESA funding (Copernicus programme) that made the presented work possible and useful discussions with colleagues from EUMETSAT. 


\section{REFERENCES}

[1] Fletcher, K., ed., [Sentinel-4: ESA's Geostationary Atmospheric Mission for Copernicus Operational Services], ESA SP-1334, 2nd edn, ESA Communications, Noordwijk, the Netherlands (2017).

[2] Grabarnik, S., Bazalgette Courrèges-Lacoste, G., Pachot, C., Gambicorti, L., Rivière, R., and Riedl, S., "In-field and Out-of-field stray light analysis for the COPERNICUS Sentinel 4 instrument”, Proc. SPIE 11180, International Conference on Space Optics — ICSO 2018, 1027-1038 (2019).

[3] Kleipool, Q., Ludewig, A., Babić, L., Bartstra, R., Braak, R., Dierssen, W., Dewitte, P.-J., Kenter, P., Landzaat, R., Leloux, J., Loots, E., Meijering, P., van der Plas, E., Rozemeijer, N., Schepers, D., Schiavini, D., Smeets, J., Vacanti, G., Vonk, F., and Veefkind, P., "Pre-launch calibration results of the TROPOMI payload on-board the Sentinel-5 Precursor satellite”, Atmos. Meas. Tech. 11(12), 6439-6479 (2018).

[4] Tol, P. J. J., van Kempen, T. A., van Hees, R. M., Krijger, M., Cadot, S., Snel, R., Persijn, S. T., Aben, I., and Hoogeveen, R. W. M., "Characterization and correction of stray light in TROPOMI-SWIR", Atmos. Meas. Tech. 11(7), 4493-4507 (2018).

[5] Mariani, F., Caron, J., Taccola, M., Grabarnik, S., and Bezy, J.-L., "StrayLux: an efficient tool for stray-light modelling in optical instruments”, Proc. SPIE 11180, International Conference on Space Optics — ICSO 2018, 2961-2968 (2019).

[6] Caron, J., Taccola, M., and Bézy, J.-L., "Towards a standardized method to assess straylight in earth observing optical instruments”, Proc. SPIE 10562, International Conference on Space Optics - ICSO 2016, 665-674 (2017).

[7] Peterson, G. L., “Analytic expressions for in-field scattered light distributions”, Proc. SPIE 5178, 184-193 (2004). 